\title{
ANTIOXIDANT AND IMMUNOMODULATORY EFFECTS OF NANO-SELENIUM ON RESPONSE OF BROILERS TO ND VACCINE
}

\author{
DALIA M. AZAB ${ }^{1}$; HEMAT S. EL-SAYED ${ }^{2}$ and AYMAN S. EL-HABBAA ${ }^{3}$ \\ ${ }^{1}$ Biochemistry Department (Pharmacology), Animal Health Research Institute, Benha-Branch, Agriculture Research \\ Center (ARC), Egypt. \\ ${ }^{2}$ Poultry Diseases Department Animal Health Research Institute, Benha-Branch, Agriculture Research Center (ARC), Egypt. \\ ${ }^{3}$ Department of Virology, Faculty of Veterinary Medicine, Benha University
}

Received: 31 March 2019; Accepted: 22 April 2019

\begin{abstract}
Trace element selenium occupies a privileged position among the natural antioxidant in addition to its ability to enhance the immune response in several species. The current challenges of supplementation of sodium selenite in poultry diets are always linked to low absorption and increased toxicity. Therefore, it is imperative to embrace new technologies like nanotechnology to get as an alternative source of traditional selenium supplementation in form of the nanoparticles with high bioavailability and low toxicity which will be positively reflected on biological activities of selenium. The present study was conducted to investigate the antioxidant and immunomodulatory effects of different concentrations of Nano-selenium on response of broilers to ND vaccination. A total of one hundred and eighty day old Cobb broilers were randomly allocated in 6 groups with each group applied to 3 replicate of 10 chicks, the first two groups were control negative groups without selenium supplementation and the remaining four groups were dietary supplemented with sodium selenite $(0.15 \mathrm{ppm})$ and Nano-selenium at concentration of $(0.15 \mathrm{ppm}),(0.075 \mathrm{ppm})$ and $(0.0375 \mathrm{ppm})$, respectively. The results showed that Nano-selenium had a superior effect compared to that of the sodium selenite either as an antioxidant or as an immunomodulatory and comparing the control negative groups. The most important results can be summarized in the following points; significant improvement in Feed conversion ratio (FCR), significant enhancement in activities of antioxidant enzymes without adverse effects on tested biochemical parameters as well as significant boosting in antibody titers against ND. In conclusion, Nano-selenium supplementation in commercial broilers diets could be an economically a promising multitask option, particularly when it comes to improving antioxidative status and boosting immune response to NDV (LaSota) vaccination in broilers.
\end{abstract}

Key words: Antioxidant; Broilers; Immunomodulatory; Nano-selenium; ND vaccine

\section{INTRODUCTION}

The metabolic processes in the body is produced by-products called the reactive species which included reactive oxygen species (ROS) and reactive nitrogen species (RNS) (Di Meo et al., 2016). The reactive species has a regulatory role in the interactive between host and pathogens including recognition of pathogens, expression and adaptation of genes as well as activation of host defense mechenism. More importantly, the higher percentages of the uncontrolled ROS generation are usually attributed to irreversible damage to proteins, lipids, carbohydrates, and nucleic acids (Aquilano et al., 2014). The control of these reactive species has an important impact on apoptosis (Gloire et al., 2006).

Corresponding author: Dr. HEMAT.S. EL-SAYED

E-mail address: hemat.elsayed@yahoo.com

Present address: Poultry Diseases Department. Animal Health Research Institute, Benha- Branch, Agriculture Research Center (ARC), Egypt.
Antioxidant defense systems that prevent damage caused by reactive oxygen species (ROS) during exposure to infections, inflammation, and stressors (Valko et al., 2007) are classified into enzymatic and nonenzymatic components. Enzymatic components include glutathione peroxidase (GSHPx), catalase, superoxide dismutase (SOD), and glutathione reductase, whereas the nonenzymatic system comprises glutathione, thioredoxin, melatonin, carotenoids, vitamin E (VitE), and vitamin C (Ivanov et al., 2016).

Selenium (Se) is one of a potent nutritional antioxidants that plays an important role in regulating reactive oxygen species (ROS). Selenium is an integral component of at least 25 selenoprotein which interfering with many metabolic pathways in the body tissues including Glutathione peroxidase (Brown and Arthur, 2001; Nazıroğlu et al., 2012 and Zhou et al., 2013). Beside its crucrial role as an antioxidant, it is consider a very important essential micronutrient that is needed for optimal immune 
responses (Bainbridge, 1976; Behne and Wolters, 1983; Surai and Dvorska, 2001; Gromer et al., 2005 and Hoffmann and Berry, 2008) and it was proven that it plays a vital role in enhancing the immune response of the body (Kim and Mahan, 2003; Ghazi et al., 2012; Ebeid et al., 2013 and Habibian et al., 2014). Moreover, selenium is a key element in several compounds that improve the response of immune system either by altering the production of certain cytokines or by strenghtening the immune cells to resist oxidative stress (Habibian et al., 2015).

Inorganic, organic and nano- selenium are the avaliable forms of selenium, the latter form has recently attracted the attention of scientific community due to its unique novel characters exihibited by the fabricated nanoparticles. Nanoselenium appears to be less toxic and more biocompatible compared to sodium selenite in addition to possessing a powerful properities including catalytic efficiency, adsorbing ability, surface activity and chemical stability (Wang et al., 2007; Zhang et al., 2008; Shi et al., 2011 and Boostani et al., 2015 and Skalickova et al., 2017). More importantly, selenium nanoparticles showed better antioxidant capability compared to that of other chemical forms in the bulk state with the advantage of reducing toxicity of selenium (wang et al., 2007).

Previous studies that carried on animals showed that selenium nanoparticles had significant effect on the activities of antioxidant enzymes compared to that of inorganic selenium $\left(\mathrm{Na}_{2} \mathrm{SeO}_{3}\right)$. Higher activity of glutathione peroxidase (GPx) was recorded in animals supplemmented with Nano-selenium in their diets (Zhang et al., 2007). Particularly in chicken, previous studies confirmed that dietary Nanoselenium supplementation could have a positive improvment in the antioxidative status of heatstressed broilers (EL-Deep et al., 2016) and it was effective in enhancing GSH-Px activities in the serum and liver tissue of Guangxi Yellow chicken (Zhou and Wang, 2011). Moreover, dietary nano-Se could enhance the antioxidant ability and oxidative stability in broilers (cai et al., 2012).

With regard to the immunomodulatory role of selenium nanoparticles that assessed in recent studies, it was shown that treatment with administered selenium nanoparticles in tumors bearing mice could be elvate IFN- $\gamma$ and IL-12 with signifcant induction of the Th1 platform of the immune response (Yazdi et al., 2012). Also selenium nanoparticles could be down regulated of mRNA gene expressions of pro-inflammatory cytokines in a dose-dependent manner as (iNOS), (IL-1), and TNF- $\alpha$, while marked increase of antiinflammatory cytokine IL-10 has been reported (Ding et al., 2010 and Wang et al., 2014).
Furthermore, dietry Nano-selenium supplementation in brolier showed immunostimulatory effect in chickens (EL-Deep et al., 2016) and appeared to improve the immunity of broiler chicks (Kadhim et al., 2018) along with huge improvement in humoral immunity that was observed in chicken fed on diet containing $0.30 \mathrm{mg} / \mathrm{kg}$ of Nano-selenium (Cai et al., 2012).

Newcastle disease (ND) is a continuing problem for poultry industry causing major economic lossess world wide. The caustive agent Newcastle disease virus (NDV) which is known as avian paramyxovirus type 1, belongs to the Avulavirus genus within the Paramyxoviridae (Alexander and Senne, 2008). Oxidative stresses in brain tissue are usually the main character associated with NDV infection in poultry, the virus decreases the activities of glutathione peroxidase (GPx) superoxide dismutase (SOD), and catalase (CAT) (Subbaiah et al., 2011 and 2015). Although vaccination aganist ND is an important strategy employed in endemic countries (Cornax et al., 2012) it does not prevent occurrence of outbreaks with vNDV nor prevents replication of challenge viruses (Choi et al., 2013; Jaganathan et al., 2015). Based on the above stated information and the benefits of nanotechnology application in poultry vaccination to boost immunity, the presented study aimed to clarify the antioxidant and immunomodulatory effects of dietary selenium nanoparticles on response of broilers to ND vaccine.

\section{MATERIALS AND METHOD}

\section{Selenium and selenium nanoparticles sources}

Sodium selenite $\left(\mathrm{Na}_{2} \mathrm{Se} \mathrm{O}_{3} .5 \mathrm{H}_{2} \mathrm{O}\right)$ was purchased from MERCK. Co, Germany. Selenium nanoparticles were prepared by using a chemical reduction of Sodium selenite with ascorbic acid followed by stabilizing the prepared nanoparticles by coating them with Dextrin according to modified method conducted by Malhotra et al. (2014). Characterization of prepared selenium nanoparticles including size, shape, morphology and crystallinity was done using different analytical tools including particle spectroscopy, Scanning electron microscope (SEM) and X-ray diffraction (XRD). The size of prepared nanoparticles used in our study was $60 \mathrm{~nm}$. Preparation of selenium nanoparticles was carried in Naqaa Foundation for Scientific Research, Technology and Development, Giza, Egypt.

\section{Preparation of experimental diets with sodium selenite and Nano-selenium}

Each of sodium selenite $0.15 \mathrm{mg} / \mathrm{kg}^{-1}$ diet and prepared selenium nanoparticles with concentration of $0.15,0.075$ and $0.0375 \mathrm{mg} / \mathrm{kg}^{-1}$ diet, respectively in dietary treated groups was dispersed in $5 \mathrm{ml}$ - 
distilled water then it was mist sprayed onto poultry feed, rotating in mixer and then air dried for 24 hour.

\section{Experimental design}

The experiment was performed according to the guidelines of Benha University, Egypt. A total of one hundred and eighty one day- old broiler chicks (Cobb, Al-Watania poultry company) were obtained from a commercial hatchery located in Qalyoubia, Egypt. The chicks were randomly divided into six groups of 30 birds per each (three replicate per group and 10 birds per replicate). After decaying of maternal antibodies at 21 days old, all birds in all groups except group no. 1 were received ND vaccination with Lasota strain via drinking water (Servac, Egypt). The dietary treatments of the current study were as following: birds in groups 1 and 2 fed on basal diet without Se supplementation; birds in group 3 fed on basal diet with $0.15 \mathrm{mg}$ of sodium selenite $\left[\mathrm{Na}_{2} \mathrm{SeO}_{3}\right]$ per kilogram of diet while birds in groups 4,5 and 6 were fed on basal diet with $0.15 \mathrm{mg}, 0.075 \mathrm{mg}$ and $0.0375 \mathrm{mg}$ of prepared selenium nanoparticles per kilogram of diet, respectively. Feed was ad-libitum for birds. The basal diet was formulated according to National Research Council (NRC, 1994) recommendations for all other nutrients to meet the nutritional requirements of growing broilers as shown in table1.

Table 1: Composition of the basal diets.

\begin{tabular}{|c|c|c|}
\hline Ingredient \% & Starter up to 21 days & Grower from 22 to 42 days \\
\hline Corn grain & 53.30 & 64.725 \\
\hline Soya bean $44 \%$ protein & 30.30 & 15.4 \\
\hline Concentrate & 10.0 & 10.0 \\
\hline Corn gluten & 2.20 & 6 \\
\hline Vegetable oil & 3.0 & 2.5 \\
\hline Di-calcium phosphate & 0.65 & 0.5 \\
\hline DL-Methionine & 0.22 & 0.025 \\
\hline L-Lysine & 0.15 & 0.3 \\
\hline Threonine & 0.135 & 0.09 \\
\hline Sodium chloride & 0.11 & 0.1 \\
\hline Lime stone & 0.05 & 0.05 \\
\hline Chemical analy & $I$ diets & Grower \\
\hline & & 22.480 \\
\hline ME ( & iet) & 3201.9 \\
\hline & & 1.01 \\
\hline Phosphor & ailable) & 0.45 \\
\hline & & 1.15 \\
\hline $\mathrm{Me}$ & & 0.5 \\
\hline Methioni & ine $(\%)$ & 0.84 \\
\hline \multicolumn{3}{|c|}{ Determination of selenium in experimental diets $(\mathrm{mg} / \mathrm{kg})$} \\
\hline Group 1 without Se supp. & 0.005 & \\
\hline Group2 without Se supp. & 0.005 & \\
\hline Group3 supp. with 0.015 inor & 0.147 & \\
\hline Group4 supp. with 0.015 Nan & 0.152 & \\
\hline Group5 supp. with 0.075 Nan & 0.071 & \\
\hline Group6 supp. with $0.0375 \mathrm{Na}$ & 0.038 & \\
\hline
\end{tabular}

\section{Supp. supplementation, Se. selenium}

The experimental diet was formulated in accordance with recommendations of National Research Council (NRC, 1994). Food Analysis Center, Faculty of veterinary medicine, Benha University performed the chemical analysis of diets.

\section{Growth Performance parameters}

Body weights and feed consumption per group were recorded weekly and the obtained values were used to calculate weight gain and Feed conversion ratio for each group expressed according to Wanger et al. (1983) as follows

$\mathbf{F C R}=$ Average feed intake $(\mathrm{g})$ bird/week Average body weight gain (g) bird/week

\section{Sampling}

Blood samples of five birds from each replicate per group were collected at weekly intervals from one day-old to day 42 of age for estimating maternal antibody and evaluating of humoral immunity on 
response to ND vaccine. For determination of serum biochemical and serum antioxidant parameters, blood samples of two birds from each replicate per group were collected at day 22 and 42 days of age. The samples were immediately transferred into sterile test tubes and serum was harvested. The serum was stored at $-20^{\circ} \mathrm{C}$ for later analysis of antioxidant and immune parameters.

\section{Biochemical parameters analysis:}

Activities of alanine aminotransferase(ALT), aspartate aminotransferase (AST) enzymes, total protein, albumin, uric acid, creatinine, total cholesterol in tested sera were determined using the spectrophotometric method (RAL, Barcelona, Spain.) and Bioanalytica test kits (Bioanalitika doo, Beograd, Serbia) as described by (Rej and Hoder, 1983). Serum globulin (G) was calculated as follows: $\mathrm{G}=$ total protein - albumin .

\section{Determination of antioxidant indices:}

The SOD, GSH-Px activities and MDA content were determined by ELISA kits produced by Nanjing Jiancheng Bioengineering Institute (Nanjing, China) according to Dalton et al. (2000).

\section{Haemagglutination inhibition assay (HI)}

The HI test was performed on tested chicken sera in V-bottomed microwell plates according to the OIE Manuel (OIE, 2012). Four HA unit of NDV antigen (Lasota, Servac, Egypt) were used. Negative and positive control sera were used to confirm the test results. The HI titer was determined as the reciprocal of the highest dilution of serum that caused total inhibition of HA activity with 4 HA unit of antigen. The test for each serum sample were repeated in duplicate. The HI titer were expressed as the reciprocal of $\log 2$. Tested sera were considered as positive sera with HI titers $\geq 4$ according to (OIE, 2012). Geometric mean titers (GMT) were calculated and cumulative mean titers (CMT) per each group were measured weekly during experimental period.

\section{Statistical analysis:}

The data were analyzed by determine the normality via Shapiro-Willk test. The differences between groups were analyzed by One-Way ANOVA followed by Duncan's multiple comparison Post Hoc tests (Duncan, 1955). The Statistical Package for Social Science (SPSS Inc. Released, 2009) (version 20.0; SPSS Inc., Chicago IL, USA) was used for performing the statistical analyses to determine difference between groups. Significance between mean values was set a statistically at $\mathrm{P}<0.05$.

\section{RESULTS}

Growth performance parameters (Body Weights and Feed conversion ratio $\mathrm{FCR}$ ):

In the first week there is no significant differences across the treatment groups. However, significant differences between groups $(\mathrm{P}<0.05)$ were observed starting from the second week (Table,2). Higher body weights were observed in group 4 which supplemented with Nano-selenium $0.15 \mathrm{ppm}$ compared with the control groups, on the other hand group 3 supplemented with sodium selenite $0.15 \mathrm{ppm}$ that showed lower body weights compared with control groups. FCR were significantly improved $(\mathrm{P}<0.05)$ in group supplemented with Nanoselenium $0.15 \mathrm{ppm}$ at weekly interval compared to control and other dietary treated groups as shown in table 3 .

Table 2: Effect of inorganic selenium and Nano-selenium supplementation on body weights in broilers.

\begin{tabular}{|c|c|c|c|c|c|c|c|}
\hline \multirow{2}{*}{ Dietary treatment } & \multicolumn{7}{|c|}{ Estimated body weights (g)/chick (mean \pm SE) } \\
\hline & $\begin{array}{c}\text { Zero } \\
\text { day }\end{array}$ & $\begin{array}{c}1 \mathrm{st} \\
\text { week }\end{array}$ & 2nd week & 3rd week & 4th week & 5 th week & 6th week \\
\hline $\begin{array}{c}\text { NVG-no } \\
\text { Selenium supp. }\end{array}$ & $\begin{array}{c}46.33 \\
\pm 0.67 a \\
\end{array}$ & $\begin{array}{c}166.67 \\
\pm 10.48 a \\
\end{array}$ & $\begin{array}{c}302.00 \\
\pm 0.67 a b \\
\end{array}$ & $\begin{array}{r}567.83 \\
\pm 11.29 b \\
\end{array}$ & $\begin{array}{c}993.1 \\
\pm 6.15 \mathrm{ab} \\
\end{array}$ & $\begin{array}{r}1296.67 \\
\pm 23.52 b \\
\end{array}$ & $\begin{array}{r}1682.33 \\
\pm 29.53 b \\
\end{array}$ \\
\hline $\begin{array}{c}\text { VG- no Selenium } \\
\text { supp }\end{array}$ & $\begin{array}{c}45.67 \\
\pm 0.33 a \\
\end{array}$ & $\begin{array}{l}164.67 \\
\pm 5.84 a\end{array}$ & $\begin{array}{c}301.00 \\
\pm 0.33 a b\end{array}$ & $\begin{array}{c}535.63 \\
\pm 10.79 \mathrm{bc} \\
\end{array}$ & $\begin{array}{c}931 \\
\pm 11.20 b \\
\end{array}$ & $\begin{array}{r}1309.7 \\
\pm 14.59 b \\
\end{array}$ & $\begin{array}{r}1629 \\
\pm 17.78 \mathrm{bc} \\
\end{array}$ \\
\hline $\begin{array}{c}\text { VG-T Sodium } \\
\text { selenite } 0.15 \text { ppm }\end{array}$ & $\begin{array}{c}47.67 \\
\pm 1.20 a\end{array}$ & $\begin{array}{l}160.67 \\
\pm 4.25 a\end{array}$ & $\begin{array}{l}274.67 \\
\pm 1.20 b\end{array}$ & $\begin{array}{c}514.33 \\
\pm 24.63 c\end{array}$ & $\begin{array}{c}942 \\
\pm 26.13 b\end{array}$ & $\begin{array}{c}1140.6 \\
\pm 22.98 c\end{array}$ & $\begin{array}{c}1590 \\
\pm 17.32 \mathrm{c}\end{array}$ \\
\hline $\begin{array}{c}\text { VG -T Nano-Se } \\
\text { 0.15 ppm }\end{array}$ & $\begin{array}{c}46.33 \\
\pm 0.33 a\end{array}$ & $\begin{array}{l}166.67 \\
\pm 1.76 a\end{array}$ & $\begin{array}{c}301.33 \\
\pm 0.33 a b\end{array}$ & $\begin{array}{l}635.53 \\
\pm 8.80 a\end{array}$ & $\begin{array}{c}1068 \\
\pm 51.62 \mathrm{a}\end{array}$ & $\begin{array}{c}1404.4 \\
\pm 22.24 a\end{array}$ & $\begin{array}{l}1823.67 \\
\pm 23.95 a\end{array}$ \\
\hline $\begin{array}{c}\text { VG -T Nano-Se } \\
\text { 0.075 ppm }\end{array}$ & $\begin{array}{c}48.00 \\
\pm 1.00 \mathrm{a}\end{array}$ & $\begin{array}{l}164.33 \\
\pm 6.12 \mathrm{a}\end{array}$ & $\begin{array}{l}324.66 \\
\pm 1.00 a\end{array}$ & $\begin{array}{c}544.17 \\
\pm 15.25 \mathrm{bc}\end{array}$ & $\begin{array}{c}899 \\
\pm 7.89 b\end{array}$ & $\begin{array}{r}1290.7 \\
\pm 19.05 \mathrm{~b}\end{array}$ & $\begin{array}{c}1869.3 \\
\pm 33.05 a\end{array}$ \\
\hline $\begin{array}{c}\text { VG-T Nano-Se } \\
\text { 0.0375 ppm }\end{array}$ & $\begin{array}{c}46.0 \\
0 \pm 1.00 \mathrm{a}\end{array}$ & $\begin{array}{l}166.33 \\
\pm 2.73 a\end{array}$ & $\begin{array}{l}294.33 \\
\pm 1.00 b\end{array}$ & $\begin{array}{c}528.83 \\
\pm 12.27 \mathrm{bc}\end{array}$ & $\begin{array}{c}918 \\
\pm 41.37 b\end{array}$ & $\begin{array}{r}1254.4 \\
\pm 41.81 b\end{array}$ & $\begin{array}{r}1820.7 \\
\pm 38.49 \mathrm{a}\end{array}$ \\
\hline
\end{tabular}

NVG, nonvaccinated group; VG, vaccinated group, T treated with; Supp. Supplementation. a,b,c, mean values with different superscripts in a column are statistically different at $(p<0.05)$ 
Table 3: Effect of inorganic selenium and Nano-selenium supplementation on feed conversion ratio (FCR) in broilers.

\begin{tabular}{|c|c|c|c|c|c|c|}
\hline \multirow{2}{*}{ Dietary treatment } & \multicolumn{6}{|c|}{ Feed conversion ratio $(\mathrm{FCR})($ mean $\pm \mathrm{SE})$} \\
\hline & 1 st week & 2nd week & 3rd week & 4 th week & 5th week & 6th week \\
\hline $\begin{array}{c}\text { NVG-no Selenium } \\
\text { supp. }\end{array}$ & $\begin{array}{c}1.26 \\
\pm 0.11 \mathrm{ab}\end{array}$ & $\begin{array}{c}2.56 \\
\pm 0.19 \mathrm{a}\end{array}$ & $\begin{array}{r}1.68 \\
\pm 0.07 \mathrm{~b}\end{array}$ & $\begin{array}{c}1.86 \\
\pm 0.05 \mathrm{ab}\end{array}$ & $\begin{array}{c}2.31 \\
\pm 0.21 \mathrm{ab}\end{array}$ & $\begin{array}{r}2.39 \\
\pm 0.09 \mathrm{a}\end{array}$ \\
\hline VG- no Selenium supp & $\begin{array}{r}1.27 \\
\mathbf{\pm 0 . 0 7} \mathbf{a b} \\
\end{array}$ & $\begin{array}{r}2.21 \\
\pm 0.13 a \\
\end{array}$ & $\begin{array}{r}1.93 \\
\pm 0.09 \mathrm{ab} \\
\end{array}$ & $\begin{array}{r}1.72 \\
\pm 0.03 b \\
\end{array}$ & $\begin{array}{r}2.26 \\
\pm 0.09 \mathrm{ab} \\
\end{array}$ & $\begin{array}{r}2.14 \\
\pm 0.10 b \\
\end{array}$ \\
\hline $\begin{array}{c}\text { VG-T Sodium selenite } \\
\text { 0.15 ppm }\end{array}$ & $\begin{array}{r}1.43 \\
\pm 0.40 a \\
\end{array}$ & $\begin{array}{r}2.60 \\
\pm 0.14 a \\
\end{array}$ & $\begin{array}{r}1.85 \\
\pm 0.10 b \\
\end{array}$ & $\begin{array}{r}1.67 \\
\pm 0.11 b \\
\end{array}$ & $\begin{array}{r}2.54 \\
\pm 0.13 a \\
\end{array}$ & $\begin{array}{r}2.40 \\
\pm 0.03 a \\
\end{array}$ \\
\hline $\begin{array}{c}\text { VG-T Nano-Se } 0.15 \\
\text { ppm }\end{array}$ & $\begin{array}{r}1.12 \\
\pm 0.02 b \\
\end{array}$ & $\begin{array}{r}1.50 \\
\pm 0.03 b\end{array}$ & $\begin{array}{r}1.37 \\
\pm 0.06 c \\
\end{array}$ & $\begin{array}{r}1.33 \\
\pm 0.13 \mathrm{c}\end{array}$ & $\begin{array}{r}1.98 \\
\pm 0.15 b \\
\end{array}$ & $\begin{array}{r}2.08 \\
\pm 0.07 b \\
\end{array}$ \\
\hline $\begin{array}{c}\text { VG -T Nano-Se } 0.075 \\
\text { ppm }\end{array}$ & $\begin{array}{c}1.28 \\
\pm 0.06 a b \\
\end{array}$ & $\begin{array}{c}1.61 \\
\pm 0.09 \mathrm{~b}\end{array}$ & $\begin{array}{r}1.68 \\
\pm 0.08 b \\
\end{array}$ & $\begin{array}{r}2.08 \\
\pm 0.09 a\end{array}$ & $\begin{array}{c}2.23 \\
\pm 0.07 \mathrm{ab} \\
\end{array}$ & $\begin{array}{r}2.12 \\
\pm 0.09 \mathrm{~b} \\
\end{array}$ \\
\hline $\begin{array}{c}\text { VG-T Nano-Se } \\
\text { 0.0375ppm }\end{array}$ & $\begin{array}{c}1.26 \\
\pm 0.02 \mathrm{ab}\end{array}$ & $\begin{array}{c}2.21 \\
\pm 0.11 \mathrm{a}\end{array}$ & $\begin{array}{r}2.17 \\
\pm \mathbf{0 . 0 5 a}\end{array}$ & $\begin{array}{c}1.85 \\
\pm 0.16 \mathrm{ab}\end{array}$ & $\begin{array}{c}2.25 \\
\pm 0.05 \mathrm{ab}\end{array}$ & $\begin{array}{r}2.09 \\
\pm 0.04 b\end{array}$ \\
\hline
\end{tabular}

NVG, nonvaccinated group; VG, vaccinated group, T treated with; Supp. Supplementation. a,b,c, mean values with different superscripts in a column are statistically different at $(\mathrm{p}<0.05)$

\begin{abstract}
Antioxidant enzymes of broilers at 22 and 42 days of age:

The results of antioxidant enzyme assays are given in Table (4). In general there was a significant $(\mathrm{P}<0.05)$ increase in GPX and SOD with significant decrease in MDA in groups treated with Nano-selenium compared to vaccinated group treated with inorganic Se.
\end{abstract}

Briefly, GPX level in examined sera at 22 days in treated group VGT2 (Nano-Se $0.15 \mathrm{ppm}$ ) showed significant increased compared to all groups. The highest activity of GPX was observed at 42 days of age in VGT2 and VGT3 (VG -T Nano-Se 0.15 and $0.075 \mathrm{ppm}$ ) respectively compared to $\mathrm{NVG}, \mathrm{VG}$, VGT1 and VGT4.

Regarding SOD activity, there was a significant increase in VGT2 and VGT3 compared to all groups at 42 days of age, while VGT2 showed the highest level with significant difference compared to other groups at 22 days of age.

The lowest level of MDA in serum appeared in VGT2 (VG -T Nano-Se $0.15 \mathrm{ppm}$ ) compared to the other groups at 42 days of age, while MDA level in VGT1 was significantly decreased compared to NVG and VG. At 22 days of age VGT1, VGT2 and VGT3 were decreased compared to NVG.

The GPX and SOD were improved in response to selenium nanoparticles 0.15 and $0.075 \mathrm{ppm}(\mathrm{P}<$ $0.05)$ compared to inorganic source of Se. The lowest MDA level in serum was observed in vaccinated group treated with Nano Se (VGT2 0.15 ppm). According to contrast comparisons, there were significant differences between sodium selenite versus selenium nanoparticles in GPX, SOD and MDA in serum. This indicates that selenium nanoparticles $(0.15 \mathrm{ppm})$ can be used instead of inorganic sources of $\mathrm{Se}(0.15 \mathrm{ppm})$ to produce higher activity level of GPX, SOD and lower MDA level in broiler chicks.

\section{Blood biochemical parameters of broilers at 22 and 42 days of age:}

Evaluation of dietary sodium selenite and Nanoselenium at different concentrations in the different groups on blood biochemical parameters are shown in Tables $(5,6)$.

Dietary supplementation of sodium selenite and different concentration of selenium nanoparticles does not change serum total protein, albumin and globulin at 22 and 42 days of age. In spite of that, the differences were significant $(\mathrm{P}<0.05)$ between the groups in serum cholesterol, AST, ALT, creatinine levels and uric acid.

AST and ALT levels in serum were decreased in birds fed Nano-selenium compared to inorganic Se (VGT1). ALT level was significantly decreased $(\mathrm{P}<0.05)$ in VGT2 compared to NVG at 22 days of age and also decreased in all groups treated with Nano-selenium compared to NVG and VGT1 at 42 days of age. AST level was significantly lower in VGT2 and VGT3 than VGT1 at 22 days of age and also lower than NVG and VG at 42 days of age. In addition, Uric acid level in VGT3 $(0.075 \mathrm{ppm})$ was decreased compared to NVG at 42 days of age, while no significant differences were showed at 22 days of age. Moreover, dietary Nano-seleniumVGT2 $(0.15 \mathrm{ppm})$ decrease the serum creatinine level compared to all groups at 22 days of age, while at 42 days of age the difference in VGT2 was decreased significantly compared to NVG, VG, and VGT3 while no significant changes were observed compared to VGT1 and VGT4. Cholesterol level was lower in VGT2 than all groups at 22 days of age, while no differences were showed compared to VGT1, but the level was decreased in VGT2 than NVG, VG, VGT3 and VGT4 at 42 days of age. 
Table 4: Antioxidant parameters of inorganic selenium and different concentrations of Nano-selenium in serum of broilers at 22 and 42 days of age (Mean* \pm SE).

\begin{tabular}{|c|c|c|c|c|c|c|}
\hline \multirow{3}{*}{ Dietary treatment } & \multicolumn{6}{|c|}{ Antioxidant parameters } \\
\hline & \multicolumn{2}{|c|}{ GPX (ng/ml) } & \multicolumn{2}{|c|}{ MDA (nmol/ml) } & \multicolumn{2}{|c|}{ SOD $(\mathbf{U} / \mathbf{L})$} \\
\hline & 22 day & 42 day & 22 day & 42 day & 22 day & 42 day \\
\hline NVG-no Selenium supp. & $\begin{array}{c}0.125 \\
\pm 0.017^{c}\end{array}$ & $\begin{array}{c}0.136 \\
\pm 0.018\end{array}$ & $\begin{array}{c}0.458 \\
\pm 0.022^{\mathrm{a}}\end{array}$ & $\begin{array}{c}0.401 \\
\pm 0.019^{a}\end{array}$ & $\begin{array}{c}0.102 \\
\pm 0.011^{c}\end{array}$ & $\begin{array}{r}0.092 \\
\pm 0.01^{\mathrm{c}}\end{array}$ \\
\hline VG- no Selenium supp. & $\begin{array}{c}0.139 \\
\pm 0.016\end{array}$ & $\begin{array}{c}0.164 \\
\pm 0.013^{c}\end{array}$ & $\begin{array}{c}0.418 \\
\pm 0.018^{\mathrm{ab}}\end{array}$ & $\begin{array}{c}0.399 \\
\pm 0.025^{a}\end{array}$ & $\begin{array}{c}0.183 \\
\pm 0.019^{b}\end{array}$ & $\begin{array}{c}0.157 \\
\mathbf{\pm 0 . 0 1 5}\end{array}$ \\
\hline $\begin{array}{l}\text { VG-T1 Sodium selenite } \\
0.15 \mathrm{ppm}\end{array}$ & $\begin{array}{c}0.185 \\
\pm 0.011^{\text {bc }}\end{array}$ & $\begin{array}{c}0.263 \\
\pm 0.015^{b}\end{array}$ & $\begin{array}{c}0.372 \\
\pm 0.035^{b}\end{array}$ & $\begin{array}{c}0.304 \\
\pm 0.026^{b}\end{array}$ & $\begin{array}{c}0.205 \\
\pm \mathbf{0 . 0 1 3}^{\mathrm{b}}\end{array}$ & $\begin{array}{c}0.299 \\
\pm \mathbf{0 . 0 1 8}^{b}\end{array}$ \\
\hline VG-T2 Nano-Se 0.15 ppm & $\begin{array}{c}0.326 \\
\pm 0.044^{\mathrm{a}}\end{array}$ & $\begin{array}{c}0.415 \\
\pm 0.012^{\mathrm{a}}\end{array}$ & $\begin{array}{c}0.339 \\
\pm 0.030^{b}\end{array}$ & $\begin{array}{c}0.231 \\
\pm 0.018\end{array}$ & $\begin{array}{c}0.335 \\
\mathbf{\pm 0 . 0 3 3}\end{array}$ & $\begin{array}{c}0.447 \\
\mathbf{\pm 0 . 0 2 3}\end{array}$ \\
\hline $\begin{array}{c}\text { VG -T3 Nano-Se } 0.075 \\
\text { ppm. }\end{array}$ & $\begin{array}{c}0.229 \pm 0 . \\
016^{b}\end{array}$ & $\begin{array}{l}0.385 \pm \\
0.021^{\mathrm{a}}\end{array}$ & $\begin{array}{c}0.350 \pm 0 . \\
021^{\mathrm{b}}\end{array}$ & $\begin{array}{c}0.272 \\
\pm 0.020^{b c}\end{array}$ & $\begin{array}{l}0.244 \pm \\
0.023^{b}\end{array}$ & $\begin{array}{c}0.432 \\
\pm 0.039^{a}\end{array}$ \\
\hline $\begin{array}{l}\text { VG-T4 Nano-Se } \\
0.0375 \mathrm{ppm} .\end{array}$ & $\begin{array}{c}0.175 \pm 0 . \\
030^{\mathrm{bc}}\end{array}$ & $\begin{array}{l}0.244 \pm \\
0.017^{b}\end{array}$ & $\begin{array}{c}0.407 \pm 0 \\
025^{\mathrm{ab}}\end{array}$ & $\begin{array}{c}0.311 \\
\pm 0.029^{b}\end{array}$ & $\begin{array}{l}0.186 \pm \\
0.027^{b}\end{array}$ & $\begin{array}{c}0.249 \\
\pm 0.027^{b}\end{array}$ \\
\hline
\end{tabular}

NVG, non-vaccinated group; VG, vaccinated group, T treated with; Supp. Supplementation. a,b,c, mean values with different superscripts in a column are statistically different at $(\mathrm{p}<0.05)$; *mean of 6 birds.

Table 5: Effect of inorganic selenium and Nano-selenium supplementation on blood biochemical parameters in broilers at 22 days of age (Mean* $*$ SE).

\begin{tabular}{|c|c|c|c|c|c|c|c|c|}
\hline \multirow[b]{2}{*}{ Dietary treatment } & \multicolumn{8}{|c|}{ Blood biochemical parameters at 22 days } \\
\hline & $\operatorname{ALT}(\mathbf{U} / \mathbf{L})$ & $\operatorname{AST}(\mathbf{U} / \mathbf{L})$ & $\begin{array}{c}\text { Total } \\
\text { protein } \\
(\mathrm{gm} / \mathrm{dl})\end{array}$ & $\begin{array}{l}\text { Albumin } \\
(\text { gm/dl) }\end{array}$ & $\begin{array}{l}\text { globulin } \\
(\mathrm{gm} / \mathrm{dl})\end{array}$ & $\begin{array}{l}\text { Uric acid } \\
(\mathbf{m g} / \mathbf{d l})\end{array}$ & $\begin{array}{c}\text { Creatinine } \\
(\mathbf{m g} / \mathrm{dl})\end{array}$ & $\begin{array}{c}\text { Total } \\
\text { Cholesterol } \\
(\mathbf{m g} / \mathbf{d l})\end{array}$ \\
\hline $\begin{array}{l}\text { NVG-no Selenium } \\
\text { supp. }\end{array}$ & $\begin{array}{c}25.19 \\
\pm 1.02 \mathrm{ab}\end{array}$ & $\begin{array}{l}178.90 \\
\pm 2.36 a\end{array}$ & $\begin{array}{c}3.82 \\
\pm 0.068 \mathrm{ab}\end{array}$ & $\begin{array}{c}1.58 \\
\pm 0.111 a\end{array}$ & $\begin{array}{c}2.24 \\
\pm 0.128 \mathrm{a}\end{array}$ & $\begin{array}{c}4.97 \\
\pm 0.432 \mathrm{ab}\end{array}$ & $\begin{array}{c}0.35 \\
\pm 0.015 a\end{array}$ & $\begin{array}{l}156.77 \\
\pm 2.83 \mathrm{a}\end{array}$ \\
\hline $\begin{array}{l}\text { VG- no Selenium } \\
\text { supp. }\end{array}$ & $\begin{array}{r}26.81 \\
\pm 1.21 \mathrm{a}\end{array}$ & $\begin{array}{l}172.70 \\
\pm 3.03 \mathrm{a}\end{array}$ & $\begin{array}{c}3.47 \\
\pm 0.18 b\end{array}$ & $\begin{array}{c}1.73 \\
\pm 0.166 a\end{array}$ & $\begin{array}{c}1.74 \\
\pm 0.338 a\end{array}$ & $\begin{array}{c}5.71 \\
\pm 0.217 \mathrm{a}\end{array}$ & $\begin{array}{c}0.32 \\
\pm 0.018 a\end{array}$ & $\begin{array}{c}156.18 \\
\pm 2.99 \mathrm{ab}\end{array}$ \\
\hline $\begin{array}{l}\text { VG-T1 Sodium } \\
\text { selenite } 0.15 \text { ppm }\end{array}$ & $\begin{array}{c}25.20 \\
\pm 0.89 \mathrm{ab}\end{array}$ & $\begin{array}{l}174.22 \\
\pm 2.24 \mathrm{a}\end{array}$ & $\begin{array}{c}3.71 \\
\pm 0.258 \mathrm{ab}\end{array}$ & $\begin{array}{c}1.64 \\
\pm 0.18 \mathrm{a}\end{array}$ & $\begin{array}{c}2.07 \\
\pm 0.341 \mathrm{a}\end{array}$ & $\begin{array}{c}4.51 \\
\pm 0.202 b\end{array}$ & $\begin{array}{c}0.34 \\
\pm 0.016 a\end{array}$ & $\begin{array}{c}148.57 \\
\pm 3.52 b c\end{array}$ \\
\hline $\begin{array}{c}\text { VG -T2 Nano-Se } \\
\text { 0.15 ppm }\end{array}$ & $\begin{array}{c}22.97 \\
\pm 0.69 b\end{array}$ & $\begin{array}{r}161.42 \\
\pm 3.25 b\end{array}$ & $\begin{array}{c}4.04 \\
\pm 0.41 \mathrm{ab}\end{array}$ & $\begin{array}{c}1.66 \\
\pm 0.128 a\end{array}$ & $\begin{array}{c}2.39 \\
\pm 0.438 \mathrm{a}\end{array}$ & $\begin{array}{c}4.16 \\
\pm 0.232 b\end{array}$ & $\begin{array}{c}0.27 \\
\pm 0.020 b\end{array}$ & $\begin{array}{l}132.30 \\
\pm 1.66 \mathrm{e}\end{array}$ \\
\hline $\begin{array}{c}\text { VG -T3 Nano-Se } \\
\text { 0.075 ppm }\end{array}$ & $\begin{array}{c}24.29 \\
\pm 1.03 \mathrm{ab}\end{array}$ & $\begin{array}{r}157.19 \\
\pm 2.98 \mathrm{~b}\end{array}$ & $\begin{array}{c}3.44 \\
\pm 0.23 b\end{array}$ & $\begin{array}{c}1.94 \\
\pm 0.219 \mathrm{a}\end{array}$ & $\begin{array}{c}1.50 \\
\pm 0.187 a\end{array}$ & $\begin{array}{c}4.29 \\
\pm 0.46 \mathrm{~b}\end{array}$ & $\begin{array}{c}0.33 \\
\pm 0.015 a\end{array}$ & $\begin{array}{l}140.13 \\
\pm 2.53 d\end{array}$ \\
\hline $\begin{array}{c}\text { VG-T4 Nano-Se } \\
\text { 0.0375ppm }\end{array}$ & $\begin{array}{c}25.56 \\
\pm 0.43 a b\end{array}$ & $\begin{array}{r}171.62 \\
\pm 3.21 \mathrm{a}\end{array}$ & $\begin{array}{c}4.39 \\
\pm 0.37 \mathrm{a}\end{array}$ & $\begin{array}{c}1.96 \\
\pm 0.131 a\end{array}$ & $\begin{array}{c}2.44 \\
\pm 0.380 a\end{array}$ & $\begin{array}{c}4.25 \\
\pm 0.38 \mathrm{~b}\end{array}$ & $\begin{array}{c}0.34 \\
\pm 0.015 a\end{array}$ & $\begin{array}{c}143.30 \\
\pm 2.29 \mathrm{~cd}\end{array}$ \\
\hline
\end{tabular}

NVG, non-vaccinated group; VG, vaccinated group, T treated with; Supp. Supplementation. a,b,c, mean values with different superscripts in a column are statistically different at $(\mathrm{p}<0.05)$; *mean of 6 birds 
Table 6: Effect of inorganic selenium and Nano-selenium supplementation on blood biochemical parameters in broilers at 42 days of age $($ Mean $* \pm \mathrm{SE})$.

\begin{tabular}{|c|c|c|c|c|c|c|c|c|}
\hline \multirow[b]{2}{*}{ Dietary treatment } & \multicolumn{8}{|c|}{ Blood biochemical parameters at 42 days } \\
\hline & $\operatorname{ALT}(\mathbf{U} / \mathbf{L})$ & $\operatorname{AST}(\mathbf{U} / \mathbf{L})$ & $\begin{array}{c}\text { Total } \\
\text { protein } \\
(\mathrm{gm} / \mathrm{dl})\end{array}$ & $\underset{(\mathrm{gm} / \mathrm{dl})}{\operatorname{Albumin}}$ & $\begin{array}{l}\text { Globulin } \\
\text { (gm/dl) }\end{array}$ & $\begin{array}{c}\text { Uric acid } \\
(\mathrm{mg} / \mathrm{dl})\end{array}$ & $\begin{array}{l}\text { Creatinine } \\
(\mathrm{mg} / \mathrm{dl})\end{array}$ & $\begin{array}{c}\text { Total } \\
\text { Cholesterol } \\
(\mathrm{mg} / \mathrm{dl})\end{array}$ \\
\hline $\begin{array}{c}\text { NVG-no Selenium } \\
\text { supp. }\end{array}$ & $\begin{array}{r}27.18 \\
\pm 0.829 a \\
\end{array}$ & $\begin{aligned} 185.13 \\
\pm 2.04 a \\
\end{aligned}$ & $\begin{array}{c}4.08 \\
\pm 0.278 a \\
\end{array}$ & $\begin{array}{c}1.96 \\
\pm 0.061 \mathrm{a} \\
\end{array}$ & $\begin{array}{c}2.13 \\
\pm 0.29 \mathrm{ab} \\
\end{array}$ & $\begin{array}{c}3.98 \\
\pm 0.347 \mathrm{a} \\
\end{array}$ & $\begin{array}{c}0.32 \\
\pm 0.016 a \\
\end{array}$ & $\begin{array}{l}159.18 \\
\pm 2.47 \mathrm{a} \\
\end{array}$ \\
\hline $\begin{array}{l}\text { VG- no Selenium } \\
\text { supp. }\end{array}$ & $\begin{array}{c}24.51 \\
\pm 0.749 \mathrm{bc}\end{array}$ & $\begin{array}{r}177.69 \\
\pm 2.05 b\end{array}$ & $\begin{array}{c}4.43 \\
\pm 0.199 \mathrm{a}\end{array}$ & $\begin{array}{c}1.90 \\
\pm 0.064 a\end{array}$ & $\begin{array}{c}2.53 \\
\pm 0.151 \mathrm{a}\end{array}$ & $\begin{array}{c}3.80 \\
\pm 0.184 a b\end{array}$ & $\begin{array}{c}0.33 \\
\pm 0.010 \mathrm{a}\end{array}$ & $\begin{array}{r}160.42 \\
\pm 2.95 \mathrm{a}\end{array}$ \\
\hline $\begin{array}{c}\text { VG-T1 Sodium } \\
\text { selenite } 0.15 \mathrm{ppm} .\end{array}$ & $\begin{array}{c}26.76 \\
\pm 0.993 \mathrm{ab}\end{array}$ & $\begin{array}{c}170.59 \\
\pm 2.28 \mathrm{~cd}\end{array}$ & $\begin{array}{c}4.49 \\
\pm 0.283 a\end{array}$ & $\begin{array}{c}1.96 \\
\pm 0.165 a\end{array}$ & $\begin{array}{c}2.52 \\
\pm 0.241 \mathrm{a}\end{array}$ & $\begin{array}{c}3.31 \\
\pm 0.213 \mathrm{ab}\end{array}$ & $\begin{array}{c}0.29 \\
\pm 0.015 \mathrm{ab}\end{array}$ & $\begin{array}{l}127.83 \\
\pm 1.77 d\end{array}$ \\
\hline $\begin{array}{c}\text { VG -T 2Nano-Se } \\
0.15 \text { ppm. }\end{array}$ & $\begin{array}{c}23.56 \\
\pm 0.702 \mathrm{c} \\
\end{array}$ & $\begin{array}{l}165.46 \\
\pm 2.32 \mathrm{~d} \\
\end{array}$ & $\begin{array}{r}4.04 \\
\pm 0.32 \mathrm{a} \\
\end{array}$ & $\begin{array}{c}2.04 \\
\pm 0.195 a \\
\end{array}$ & $\begin{array}{c}1.99 \\
\pm 0.363 \mathrm{ab} \\
\end{array}$ & $\begin{array}{c}3.85 \\
\pm 0.402 \mathrm{ab} \\
\end{array}$ & $\begin{array}{c}0.25 \\
\pm 0.018 b \\
\end{array}$ & $\begin{array}{l}128.87 \\
\pm 1.48 d \\
\end{array}$ \\
\hline $\begin{array}{c}\text { VG -T3 Nano-Se } \\
\text { 0.075 ppm. }\end{array}$ & $\begin{array}{r}22.39 \\
\pm 0.642 c \\
\end{array}$ & $\begin{array}{c}175.64 \\
\pm 2.36 \mathrm{bc} \\
\end{array}$ & $\begin{array}{c}3.55 \\
\pm 0.350 \mathrm{a} \\
\end{array}$ & $\begin{array}{c}2.15 \\
\pm 0.231 a \\
\end{array}$ & $\begin{array}{c}1.41 \\
\pm 0.394 a \\
\end{array}$ & $\begin{array}{c}2.99 \\
\pm 0.171 b \\
\end{array}$ & $\begin{array}{c}0.30 \\
\pm 0.017 \mathrm{a} \\
\end{array}$ & $\begin{array}{r}135.3 \\
\pm 1.91 \mathrm{c} \\
\end{array}$ \\
\hline $\begin{array}{c}\text { VG-T4 Nano-Se } \\
\text { 0.0375ppm. }\end{array}$ & $\begin{array}{c}24.21 \\
\pm 0.778 c\end{array}$ & $\begin{array}{c}180.24 \\
\pm 2.39 \text { ab }\end{array}$ & $\begin{array}{c}4.16 \\
\pm 0.294 a\end{array}$ & $\begin{array}{c}1.89 \\
\pm 0.129 a\end{array}$ & $\begin{array}{c}2.27 \\
\pm 0.359 \mathrm{ab}\end{array}$ & $\begin{array}{c}3.40 \\
\pm 0.202 \mathrm{ab}\end{array}$ & $\begin{array}{c}0.29 \\
\pm 0.015 a b\end{array}$ & $\begin{array}{l}146.50 \\
\pm 1.78 \mathrm{~b}\end{array}$ \\
\hline
\end{tabular}

NVG, non-vaccinated group; VG, vaccinated group, T treated with; Supp. Supplementation. a,b,c, mean values with different superscripts in a column are statistically different at $(\mathrm{p}<0.05)$; *mean of 6 birds,

Immunomodulatory effect of different dietary treatments on $\mathrm{HI}$ titers against ND vaccination.

In the present study we evaluate the effect of dietary Nano-selenium at different concentrations in the different groups regarding response to ND vaccination in broiler chicks by $\mathrm{HI}$ test. Our results revealed that, supplementation of Nano-selenium with concentration of $0.15 \mathrm{ppm}$ and $0.075 \mathrm{ppm}$ in groups 4 and 5, respectively showed significantly boost $(\mathrm{P}<0.05)$ in their HI titers compared to that of vaccinated control group (2) at weekly interval post vaccination. On the other hand group 3 which supplemented with inorganic selenium showed no boost effect (figure 1).

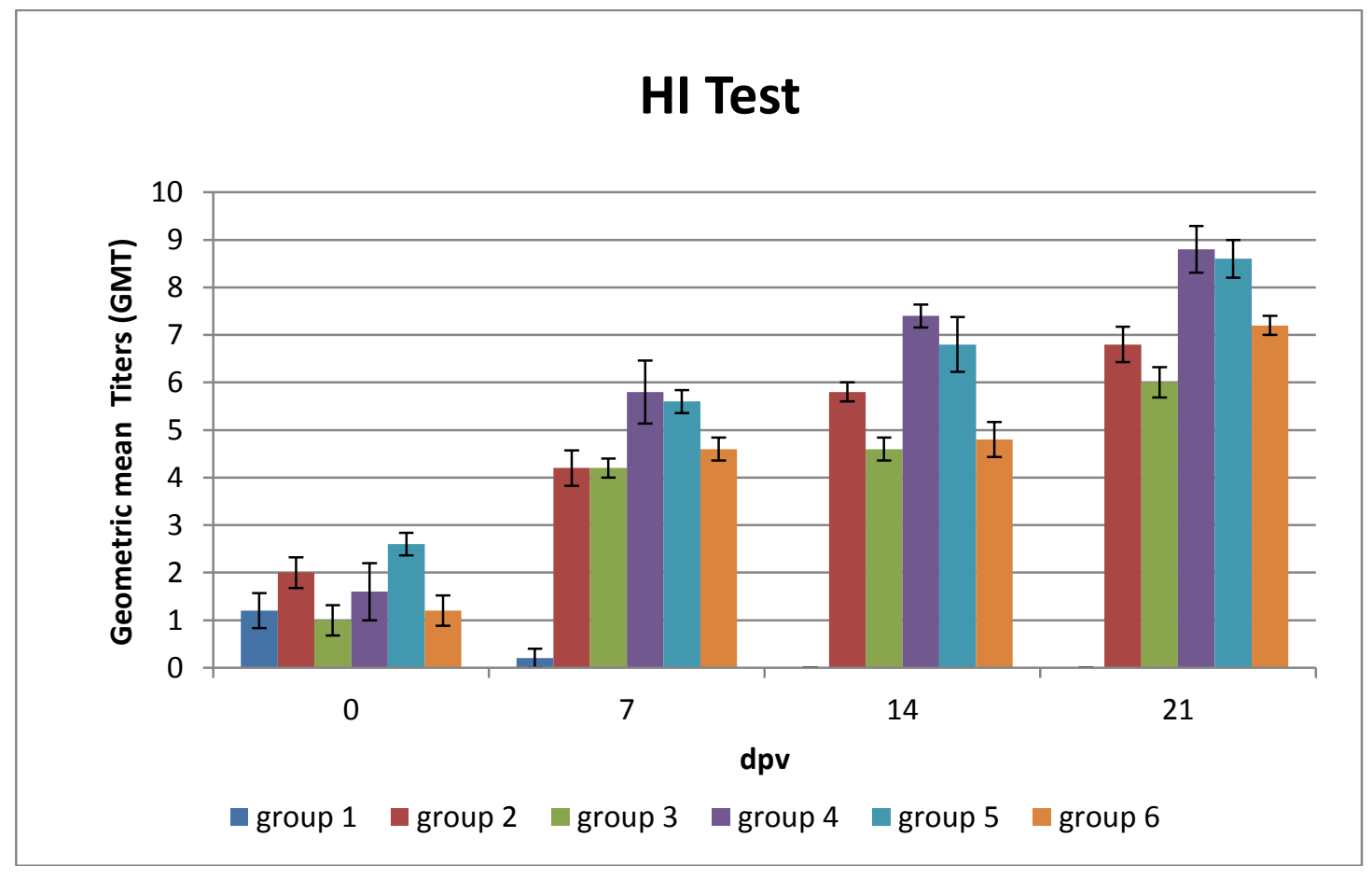

Fig. 1: Effect of inorganic selenium and Nano-selenium supplementation on antibody titers against ND vaccination in broiler chicks.

dpv, days post vaccination, group1, non-vaccinated non treated, group 2 vaccinated non treated, group 3, vaccinated dietary treated with inorganic selenium $(0.15 \mathrm{ppm})$, group 4 vaccinated dietary treated with Nanoselenium $(0.15 \mathrm{ppm})$, group 5 vaccinated dietary treated with Nano-selenium $(0.075 \mathrm{ppm})$, group 6 vaccinated dietary treated with Nano-selenium $(0.0375 \mathrm{ppm})$. 


\section{DISCUSSION}

Currently, the supplementation of poultry ration with selenium is limited to the inorganic form (sodium selenite) or selenium containing organic compound (Mohapatra et al., 2014). Following the results of several studies that confirmed the higher bioavailability and less toxicity of Nano-selenium compared to sodium selenite in different animal species including mice, rat, goat and broilers (Jia et al., 2005; Zhang et al., 2005, Wang et al., 2007; Shi et al., 2011 and Zhou and Wang, 2011). Scientists were encouraged to study the impact of dietary Nano-selenium as a good alternative choice for other traditional sources of bulk selenium used in poultry ration. The most previous studies associated with supplementation of Nano-selenium in broilers diets showed that there is no significant effect of Nano-selenium on growth performance and feed conversion (Cai et al., 2012, EL-Deep et al., 2016 and Gangadoo et al., 2017). Indeed, our results clearly indicated that body weights and FCR were significantly improved $(\mathrm{P}<0.05)$ when broiler birds were given diets supplemented with Nano-selenium $0.15 \mathrm{ppm}$ compared to other dietary treatments which showed no significant improvement in comparison with control groups. These results agreed with those obtained by Zhou and Wang, (2011) who reported that, FCR was significantly improved when birds were given diets with NanoSe. Also, Jayanthi et al. (2018) reported that, improvement of growth performance of broilers with reduction in production cost was achieved when broilers were fed on a diet containing $0.11 \mathrm{mg} / \mathrm{kg}$ of Nano-Se (75\% of inorganic Se). In our study Nanoselenium at concentration $(0.15 \mathrm{ppm})$ could improve the body weights and FCR compared to the same concentration of inorganic selenium which failed to improve the growth performance parameters. These differences might be attributed to the high absorption of Nano-selenium from the intestinal lumen into the body compared to that of inorganic selenium (sodium selenite) (Hu et al., 2012). These results agreed with EL-Deep et al. (2016) who showed the superiority of Nano-selenium compared to sodium selenite in broiler chickens regarding its efficacy and bioavailability.

Animal nutrition is important factor in animal antioxidant system. The up-regulation process of Secontaining antioxidant enzymes and glutathione pool in the body were greatly affected by selenium supplementation in animal diets (Jiang et al., 2009). Some literatures referred to the role of selenium in activating GSH-Px was reflected positively on the improvement of the antioxidant status (Ebeid et al., 2013). Both of Glutathione peroxidase and superoxide dismutase are considered an essential antioxidants that play an important role in combating toxic oxygen reduction metabolites (Zhang et al.,
2014). Herein, one of the important points in our results is the significant $(\mathrm{p}<0.05)$ increase in GPX and SOD activities in group supplemented with Nano-selenium $0.15 \mathrm{mg} / \mathrm{kg}$ diets compared to those supplemented with sodium selenite with the same concentration. These results agree with Aparna and Karunakaran (2016) who observed an increase in SOD and glutathione peroxidase cellular activity in birds fed with $(0.1875 \mathrm{mg} / \mathrm{kg})$ selenium nanoparticles when compared to control group and other treatment groups. Also Cai et al. (2012) showed an increase in activity of glutathione peroxidase in both serum and tissue in groups supplemented with Nano-selenium compared to the control group.

Oxidative stress marker, Malondialdehyde is one of the final products of cell polyunsaturated fatty acid peroxidation. In a previous study carried by Gawel et al. (2004), they showed that organic selenium can decrease the level of MDA as well as decrease the production of lipid peroxidation products compared to that of sodium selenite and they attributed to the high bioavailability of organic selenium. In the present study, MDA levels in serum were significantly decreased in vaccinated group supplemented with Nano-selenium (0.15ppm) compared to the same concentration of sodium selenite $(0.15 \mathrm{ppm})$. These results agreed with ElDeep et al. (2016) who showed that Nano-selenium in broiler diets could enhance the activities of GSHPx, SOD and CAT in addition to reducing MDA content in the liver and muscles of broilers.

Referring to the results of biochemical parameters tested in broilers sera in our study, both sodium selenite and Nano-selenium had no effect on serum total protein, albumin and globulin compared to control groups. These results agreed with those recorded by Yang et al. (2012). ALT and AST enzymes currently used as indicators of the oxidative damage of liver. The reduction in the levels of ALT, AST, urea and creatinine enhance the protection odds against oxidative damage. Moreover, higher blood cholesterol level was used as an index for stressful situations (Boostani et al., 2015). In this study, dietary Nano-Se (0.15 and $0.075 \mathrm{ppm})$ significantly decreased the levels of ALT, AST, urea, cholesterol and creatinine in broilers sera. These results supported by findings obtained by Dalia et al. (2017) who reported reduction of AST, ALT and creatinine levels in sera of broilers fed on Nano-selenium in their diets. Moreover, our results agreed with Mohapatra et al. (2014) who reported that Nano-selenium in diets of layer chicks could significantly decrease $(\mathrm{P}<0.05)$ the levels of total cholesterol and triglyceride. Our results indicated that Nano-selenium had a broad range of safety compared to that of sodium selenite. These findings 
agreed with the results of previous studies carried on broilers that referred to the low toxicity and higher bioavailability of Nano-selenium compared to sodium selenite (Hu et al., 2012).

$\mathrm{ND}$ is one of the most important diseases causing high economic losses in poultry sectors. Vaccination against ND is a routine and regular regime applied in all chicken farms in Egypt to provide the protection against the disease. Vaccine failure is one of common recurring problems in commercial poultry flocks, among the different reasons of vaccine failure which occupy the forefront is the nutritional cause where it provide the optimum condition for development and function of immune system (Khan et al., 1993). The dietary selenium is an effective element needed for optimum development and function of the immune system (Koski and Marilyn, 2003). Humoral immunity is greatly influenced by insufficient concentration of selenium in diets where low levels of $\operatorname{IgG}$ and $\operatorname{IgM}$ antibodies associated with selenium deficiency were recorded in some studies (Suchý et al., 2014). Referring to the results of previous study conducted by Funari et al. (2012) that showed there is no effect of selenium in both available forms (in- organic and organic) on humoral immunity of broilers on the response against Newcastle vaccine. Our results in the present study agreed with those obtained by Funari et al. (2012) at particular point which is: the inorganic selenium $\mathrm{Na}_{2} \mathrm{SeO}_{3}$ in vaccinated treated group 3 showed no effect on improvement of humoral immunity of broilers against the vaccine of Newcastle disease. More importantly results in our study showed that, dietary treatments with selenium nanoparticles at concentration of $0.15 \mathrm{ppm}$ and $0.075 \mathrm{ppm}$ in treated groups 3 and 4 , respectively showed significant improvement $(\mathrm{P}<0.05)$ in the humoral immunity of broiler against the vaccine of Newcastle disease at 7,14 and 21 days post vaccination compared to vaccinated non treated group (2). These results agree with those of Cai et al. (2012) who reported that, dietary supplementation of Nano-Se in broiler ration resulted in improving the humoral immunity. Moreover they found that the levels of $\operatorname{IgG}$ and $\operatorname{IgM}$ $(\mathrm{P}<0.01)$ were highest in broilers fed on diet supplemented with $0.30 \mathrm{mg} / \mathrm{kg}$ of Nano-Se, while our study found that, $0.15 \mathrm{mg} / \mathrm{kg}$ and $0.075 \mathrm{mg} / \mathrm{kg}$ of Nano-Se exhibited the highest antibody titer at weekly interval post vaccination $(\mathrm{P}<0.05)$ which was a good opportunity to reduce the economic cost of Nano-selenium. Another point of interest, selenium nanoparticles possess superior effect on humoral immunity in comparison to inorganic selenium $\left(\mathrm{Na}_{2} \mathrm{SeO}_{3}\right)$ and this was an excellent chance to take advantage of selenium nanoparticles with reduced selenium toxicity.

\section{CONCLUSION}

Incorporation of new technologies applications particularly nanotechnology could be a promising solution for current challenges in poultry nutrition. The present study suggested that Nano-Se could be used in broilers ration as an alternative source instead of traditional sources of selenium. Our results showed that Nano-selenium could improve growth performance and immunity as well as enhance the antioxidative status in broilers.

\section{CONFLICT OF INTEREST STATEMENT}

The authors declare no conflict of interest.

\section{ACKNOWLEDGEMENT}

The authors highly thankful to Dr. Wesam A. Tawfik, the head of Naqaa foundation for scientific research, technology and development for her help and support in preparation and characterization of Nano-selenium.

\section{REFERENCES}

Alexander, D.J. and Senne, D.A. (2008): Newcastle disease, other avian paramyxoviruses, and pneumovirus infections. In: Diseases of poultry, ed. Y. M. Saif, ed. Blackwell, Ames, IA. pp. 75 .

Aquilano, K.; Baldelli, S. and Ciriolo, M.R. (2014): Glutathione: New roles in redox signaling for an old antioxidant. Front. Pharmacol. 5, 196.

Aparna, N. and Karunakaran, R. (2016): Effect of Selenium Nanoparticles Supplementation on Oxidation Resistance of Broiler Chicken. Indian Journal of Science and Technology, Vol 9(S1). DOI:10.17485/ijst/2016/v9iS1/ 106334.

Bainbridge, D.R. (1976): Use of (75Se) LSelenomethionine as a label for lymphoid cells. Immunology. 30:135-144.

Behne, D. and Wolters, W. (1983): Distribution of selenium and glutathione peroxidase in the rat. J Nutr. 113:456-461.

Boostani, A.; Sadeghi, A.A.; Mousavi, S.N.; Chamani, M. and Kashan, N. (2015): The Effects of Organic, Inorganic, and NanoSelenium on Blood Attributes in Broiler Chickens Exposed to Oxidative Stress. Acta Scientiae Veterinariae, 43: 1264.

Brown, K.M. and Arthur, J.R. (2001): Selenium, selenoproteins and human health: a review. Public Health Nutr. 4:593-599.

Cai, S.J.; Wu, C.X.; Gong, L.M.; Song, T.; Wu, H. and Zhang, L.Y. (2012): Effects of nanoselenium on performance, meat quality, immune function, oxidation resistance, and 
tissue selenium content in broilers. Poultry Science. 91(10): 2532-2539.

Choi, K.S.; Kye, S.J.; Kim, J.Y.; Damasco, V.R.; Sorn, S. and Lee, Y.J. (2013): Molecular epidemiological investigation of velogenic Newcastle disease viruses from village chickens in Cambodia. Virus Genes.; 47(2): 244-249.

Cornax, I.; Miller, P.J. and Afonso, A.C.L. (2012): Characterization of Live LaSota Vaccine Strain-Induced Protection in Chickens Early Challenge with a Virulent Newcastle Disease Virus of Heterologous Genotype. Avian Diseases. 56:464-470.

Dalia, A.M.; Loh, T.C.; Sazili, A.Q. ; Jahromi, M.F. and Samsudin, A.A. (2017): The effect of dietary bacterial organic selenium on growth performance, antioxidant capacity, and Selenoproteins gene expression in broiler chickens. BMC Vet Res.; 13: 254.

Dalton, T.; Dieter, M.Z.; Yang, Y.; Shertzer, H.G. and Nebert, D.W. (2000): Knockout of the mouse glutamate cysteine ligase catalytic subunit (Gclc) gene: embryonic lethal when homozygous, and proposed model for moderate glutathione deficiency when heterozygous. Biochem Biophys Res Commun. 20; 279 (2): 324-329.

Di Meo, S.; Reed, T.T.; Venditti, P. and Victor, V.M. (2016): Role of ROS and RNS sources in physiological and pathological conditions. Oxid. Med. Cell. Longev. 2016, 1245049.

Ding, H.; Zhou, M.; Zhang, R.P. and Xu, S.L. (2010): Ganoderma lucidum extract protects dopaminergic neurons through inhibiting the production of inflammatory mediators by activated microglia. Sheng Li Xue Bao. 62:547-554.

Duncan, B.D. (1955): Multiple range and multiple ftest. Biometrics. 11:1-42.

Ebeid, T.A.; Zeweil, H.S.; Basyony, M.M.; Dosoky, W.M. and Badry, H. (2013): Fortification of rabbit diets with vitamin $\mathrm{E}$ or selenium affects growth performance, lipid peroxidation, oxidative status and immune response in growing rabbits. Livestock Science. 155: 323-331.

El-Deep, M.H.; Ijiri, D.; Ebeid, T.A. and Ohtsuka, A. (2016): Effects of Dietary Nano-Selenium Supplementation on Growth Performance, Antioxidative Status, and Immunity in Broiler Chickens under Thermoneutral and High Ambient Temperature Conditions. The Journal of Poultry Science. October 2016, doi: 10.2141/jpsa.0150133.

Funari, J.R.P.; De Albuquerque, R.; Murarolli, V.D.A.; Raspantini, L.E.R.; Cardoso, A.L.S.P.; Tessari, E.N.C. and Alves, F.R. (2012): Different sources and levels of selenium on humoral immunity of broiler chickens. Ciência Rural. 42, 154-159. (in Portuguese)

Gangadoo, S.; Dinev, I.; Chapman, J.; Hughes, R.J; Hao Van, T.T.; Moore, R.J. and Stanley, D. (2017): Selenium nanoparticles in poultry feed modify gut microbiota and increase abundance of Faecalibacterium prausnitzii. Applied Microbiology and Biotechnology. https://doi.org/10.1007/s00253-017-8688-4.

Gawet, S.; Wardas, M.; Niedworok, E. and Wardas, $P$. (2004): Malondialdehyde (MDA) as a lipid peroxidation marker. Wiadomosci lekarskie (Warsaw, Poland) 57(9-10):453455.

Ghazi, S.; Habibian, M.; Moeini, M.M. and Abdolmohammadi, A. (2012): Effects of dietary selenium, vitamin $\mathrm{E}$, and their combination on growth, serum metabolites, and antioxidant defense system in skeletal muscle of broilers under heat stress. Biol Trace Elem Res. 148: 322-330.

Gloire, G.; Legrand-Poels, S. and Piette, J. (2006): NF-KappaB activation by reactive oxygen species: Fifteen years later. Biochem. Pharmacol. 72, 1493-1505.

Gromer, S.; Eubel, J.K.; Lee, B.L. and Jacob, J. (2005): Human selenoproteins at a glance. Cell Mol Life Sci. 62:2414-2437.

Habibian, M.; Ghazi, S.; Moeini, M.M. and Abdolmohammadi, A. (2014): Effects of dietary selenium and vitamin $\mathrm{E}$ on immune response and biological blood parameters of broilers reared under thermoneutral or heat stress conditions. Int $\mathrm{J}$ Biometeorol 58: 741-752.

Habibian, M.; Sadeghi, G.; Ghazi, S. and Moeini, M.M. (2015): Selenium as a Feed Supplement for Heat-Stressed Poultry: a Review. Biol Trace Elem Res. 165: 183-193. DOI 10.1007/s12011-015-0275-x.

Hoffmann, P.R. and Berry, M.J. (2008): The influence of selenium on immune responses. Mol Nutr Food Res. November; 52(11): 1273-1280. doi:10.1002/mnfr.200700330.

Hu, H.C.; Li, Y.L.; Xiong, L.; Zhang, H.M.; Song, J. and Xia, M.S. (2012): Comparative effects of nano elemental selenium and sodium selenite on selenium retention in broiler chickens. Animal Feed Science and Technology. 177: 204-210.

Ivanov, A.V.; Valuev-Elliston, V.T.; Ivanova, O.N.; Kochetkov, S.N.; Starodubova, E.S.; Bartosch, B. and Isaguliants, M.G. (2016): Oxidative stress during HIV infection: Mechanisms and consequences. Oxid. Med. Cell. Longev. 2016, 8910396.

Jaganathan, S.; Ooi, P.T.; Phang, L.Y.; Allaudin, Z.N.; Yip, L.S. and Choo, P.Y. (2015): Observation of risk factors, clinical manifestations and genetic characterization of 
recent Newcastle Disease Virus outbreak in West Malaysia. BMC Vet Res. 11: 219.

Jai, X.; Li, N. and Chen, J. (2005): A Subchronic toxicity study of elemental Nano-Se in Sprague-Dawley rats. Life Sci. 76: 19892003.

Jayanthi, K.; Kumanan1, K.; Om Prakash, A.V.; Vijayarani, K. and Ramesh, J. (2018): Cost Effective Inclusion of Nano Selenium in Broiler Feed for Their Performance. Indian Vet. J. May 2018, 95 (05): 55 - 57.

Jiang, Z.; Lin, Y.; Zhou, G.; Luo, L.; Jiang, S. and Chen, F. (2009): Effects of dietary selenomethionine supplementation on growth performance, meat quality and antioxidant property in yellow broilers. J Agric Food Chem. 57(20):9769-9772.

Kadhim, Z.M. (2018): Study the effect of adding two levels of organic selenium and nano-selenium particles to the ration on some blood traits and lymphatic organs of broiler chicks (Ross 308). M.V.SC. Thesis, Animal Production Department, College of Agriculture, AlQasim Green University, Iraq.

Khan, S.A.; Iqbal, M. and Ashraf, S.K. (1993): Poultry industry in Pakistan. Agro. Vet. News. 5(6): 9.

Kim, Y.Y and Mahan, D.C. (2003): Biological aspects of Selenium in farm animals. Asian Aust. J. Anim. Sci. 16: 435-444.

Koski, K.G. and Marilyn, E. (2003): Gastrointestinal nematodes, trace elements, and immunity. J. Trace Elem. Exp. Med. 16: 237-251.

Malhotra, S.; Jha, N. and Desai, K. (2014): A Superficial synthesis of selenium Nanospheres using wet chemical approach. International Journal of Nanotechnology and Application (IJNA) . Vol. 3, Issue 4, 7-14.

Mohapatra, P.; Swain R.K.; Behera, M.T.; Swain, S.S.; Behura, N.C.; Sabat, S.C.; Sethv, K.; Dhama, K. and Javasankar, P. (2014): Effect of Dietary Nano-Selenium on Tissue Deposition, Antioxidant Status and Immune Function in Layer Chicks. International Journal of Pharmacology. 10(3): 160-167. DOI:10.3923/ijp.2014.160.167.

National Research Council (NRC). (1994): Nutrient requirement of poultry, 9th rev. ed. National Academy press. Washington, DC. USA.

Nazıroğlu, M.; Yıldız, K.; Tamtürk, B.; Erturan, I. and Flores-Arce, M. (2012): Selenium and psoriasis. Biol Trace Elem Res 150: 3-9.

OIE (2012): Newcastle disease. Pages 555-573 in Manual of Diagnostic Tests and Vaccines for Terrestrial Animals. 7th ed. World Organisation for Animal Health (OIE), Paris, France.

Rej, R. and Hoder, M. (1983): Aspartate aminotransferase. Pages 416-433 in Methods of Enzymatic Analysis. 3rd ed. H. U.
Bergmeyer, J. Bergmeyer, and M. Grassl. Verlag- Chemie, Weinheim, Germany.

Skalickova, S.; Milosavljevic, V.; Cihalova, K.; Horky, P.; Richtera, L. and Adam, V. (2017): Selenium nanoparticles as a nutritional supplement. Nutrition 33:83-90.

Snedecor, G.W. and Cochran, W.C. (1989): Statistical methods. The eighth. Edition, Iowa University Press, Ames, Iowa, USA.

SPSS Inc. Released (2009): PASW Statistics for Windows, Version 18.0. Chicago: SPSS Inc.

Subbaiah, K.C.; Raniprameela, D.; Visweswari, G.; Rajendra, W. and Lokanatha, V. (2011): Perturbations in the antioxidant metabolism during newcastle disease virus (NDV) infection in chicken: Protective role of vitamin E. Naturwissenschaften. 98, 10191026.

Subbaiah, K.C.; Valluru, L.; Rajendra, W.; Ramamurthy, C.; Thirunavukkarusu, C. and Subramanyam, $R$. (2015): Newcastle disease virus (NDV) induces protein oxidation and nitration in brain and liver of chicken: Ameliorative effect of vitamin E. Int. J. Biochem. Cell Biol. 64, 97-106.

Suchý, P.; Straková, E. and Herzig, I. (2014): Selenium in poultry nutrition: a review. Czech J Anim Sci 59: 495-503.

Surai, P.F. and Dvorska, J.E. (2001): Is organic selenium better than inorganic sources. Feed Mix 9: 8-10.

Valko, M.; Leibfritz, D.; Moncol, J.; Cronin, M.T.; Mazur, M. and Telser, J. (2007): Free radicals and antioxidants in normal physiological functions and human disease. International Journal of Biochemistry and Cell Biology; 39(1): 44-84.

Wang, H.; Zhang, J. and Yu, H.Q. (2007): Elemental selenium at nano size possesses lower toxicity without compromising the fundamental effect on selenoenzymes: comparison with selenomethionine in mice. Free Radic Biol Med 42(10): 1524-1533.

Wang, J.; Zhang, Y.; Yuan, Y. and Yue, T. (2014): Immunomodulatory of selenium nanoparticles decorated by sulfated Ganoderma Lucidum polysaccharides. Food Chem Toxicol. 68: 183-189.

Wanger, D.D.; Furrow, R.D. and Bradly, A. (1983): Subchronic toxicity of growth promoters in broiler chickens. Ve. Pathol. 20: 353-359.

Yang, Y.; Meng, F.; Wang, P.; Jiang, Y.; Yin, Q. and Chang, J. (2012): Effect of organic and inorganic selenium supplementation on growth performance, meat quality and antioxidant property of broilers. Afr J Biotechnol. 11(12): 3031-3036.

Yazdi, M.H.; Mahdavi, M; Varastehmoradi, B.; Faramarzi, M.A. and Shahverdi, A.R. (2012): The immunostimulatory effect of biogenic selenium nanoparticles on the 4T1 breast 
cancer model: an in vivo study. Biol Trace Elem Res. 149: 22-28.

Zhang, H.; Xia, M. and Hu, C. (2007): Effect of nano-selenium on the activities of glutathione peroxidase and type-I deiodinase in the liver of weanling pigs. Sheng Wu Yi Xue Gong Cheng Xue Za Zhi. 24(1): 153-156.

Zhang, J.; Wang, X. and Xu, T. (2008): Elemental selenium at nano size (Nano-Se) as a potential chemopreventive agent with reduced risk of selenium toxicity: comparison with Se-methylselenocysteine in mice. Toxicol Sci.;101(1): 22-31.

Zhang, J.S.; Wang, X.X.Y. and Zhang, L. (2005): Comparison of short-term toxicity between Nano-Se and selenite in mice. Life Sci. 76; 1099-1109.
Zhang, L.; Wang, Y.; Zhou, Y.; Zheng, L.; Zhan, X. and $P u, Q$. (2014): Different sources of maternal selenium affect selenium retention, antioxidant status, and meat quality of 56day-old offspring of broiler breeders. Poult Sci. 93(9):2210-2219.

Zhou, J.; Huang, K. and Lei, X.G. (2013): Selenium and diabetes - evidence from animal studies. Free Radic Biol Med. 65: 1548-1556.

Zhou, X. and Wang, Y. (2011): Influence of dietary nano elemental selenium on growth performance, tissue selenium distribution, meat quality, and glutathione peroxidase activity in Guangxi Yellow chicken. Poultry Science 90: 680-686.DOI: 10.3382/ps.201000977.

\section{التأثيرات المضادة للأكسدة و المناعية للنانوسيلنيوم على استجابة دجاج التسمين للقاح النيوكاسل \\ داليا محفوظ عزب ، همت شفيق السبي ، أبين سعيد الهباء \\ E-mail: hemat.elsayed@yahoo.com Assiut University web-site: www.aun.edu.eg}

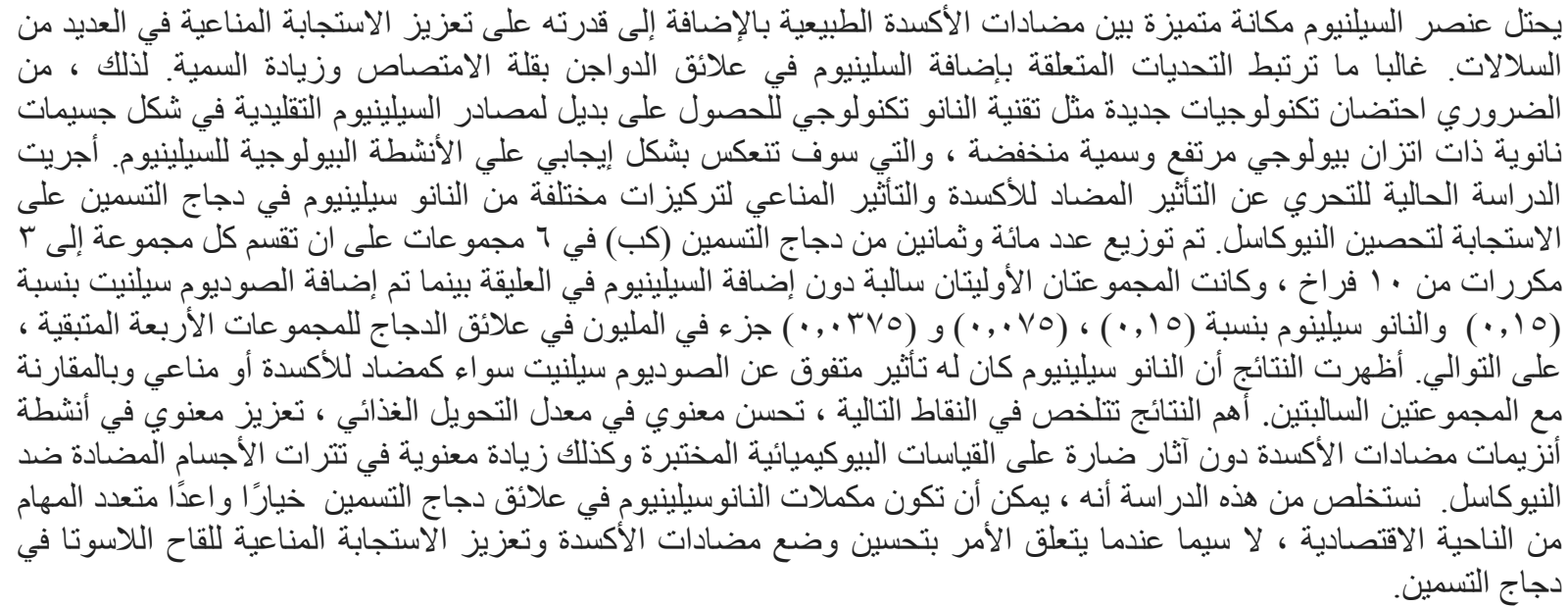

